\title{
Endoscopic hematoma evacuation for acute and subacute subdural hematoma in elderly patients
}

\author{
Kimihiko Yokosuka, MD, PhD, Masaaki Uno, MD, PhD, Kohei Matsumura, MD, Hiroki Takai, MD, \\ Hirotaka Hagino, MD, Nobuhisa Matsushita, MD, PhD, Hiroyuki Toi, MD, and \\ Shunji Matsubara, MD, PhD
}

Department of Neurosurgery, Kawasaki Medical School, Kurashiki, Okayama, Japan

\begin{abstract}
OBJECT Endoscopic surgery was performed for acute or subacute subdural hematoma (SDH), and its effectiveness and safety in elderly patients were evaluated.

METHODS Between September 2007 and November 2013, endoscopic surgery was performed in 11 elderly patients with acute SDH (8 patients) and subacute SDH (3 patients). The criteria for surgery were as follows: 1 ) the presence of clinical symptoms; 2) age older than 70 years; 3 ) no brain injury (intracerebral hematoma, brain contusion); 4) absence of an enlarging $\mathrm{SDH}$; and 5) no high risk of bleeding. Hematoma evacuation was performed with a 4-mm rigid endoscope with $\mathrm{a} 0^{\circ}$ lens and a malleable irrigation suction cannula.
\end{abstract}

RESULTS Endoscopic surgery was performed under local anesthesia. The mean age of the patients was 82.6 years (range 73-91 years). There were 5 female and 6 male patients. The mean preoperative Glasgow Coma Scale score was 12 , and 5 patients had been receiving antithrombotic drug therapy. The mean operation time was 85 minutes. Only 1 patient had rebleeding, and reoperation with the same technique was performed uneventfully in this individual. A total of 7 patients had a good recovery (modified Rankin Scale Score 0-2) at discharge.

CONCLUSIONS Endoscopic hematoma evacuation of acute and subacute SDH is a safe and effective method of clot removal that minimizes operative complications. This technique may be a less invasive method for treating elderly patients with acute and subacute SDHs.

http://thejns.org/doi/abs/10.3171/2014.10.JNS14915

KEY WORDS acute subdural hematoma; elderly patients; endoscopic hematoma evacuation; subacute subdural hematoma; surgical technique

$\Lambda$ $\mathrm{s}$ the population of Japan ages, less invasive operations are becoming mainstream. For patients with acute and subacute subdural hematomas (SDHs), a large craniotomy or craniectomy used to be performed, and morbidity and mortality rates were high because of invasive surgery, long operating time, much blood loss, and the risks of infection and general anesthesia. ${ }^{15}$ Endoscopic hematoma evacuation of a spontaneous cerebral hemorrhage is a minimally invasive and safe technique. ${ }^{8}$ Therefore, we hypothesized that endoscopic surgery was less invasive and more effective for elderly patients with acute and subacute SDHs. The efficacy of the endoscopic hematoma evacuation technique for these patients and their outcomes are reported.

\section{Methods}

Between September 2007 and November 2013, 11 patients with acute ( 8 patients) and subacute (3 patients) SDHs who underwent endoscopic hematoma evacuation surgery were analyzed. An SDH was classified as follows: acute (time from onset up to 3 days) and subacute (time of onset from 4 to 20 days). ${ }^{10}$ The demographic and clinical characteristics of these patients are shown in Table 1. There were 6 male and 5 female patients, ranging in age from 73 to 91 (average 82.6) years. Initial head CT demonstrated an SDH in all cases. The patients' preoperative Glasgow Coma Scale (GCS) scores ranged from 6 to 15 (average 12). The causes of SDH included 5 falls, 1 act of violence, 1 that occurred after bur hole surgery, and 4 unknown.

In our department, a large craniotomy or craniectomy is frequently the first-choice treatment for acute and subacute SDHs. Endoscopic hematoma evacuation was performed for acute and subacute SDH in carefully selected patients. The indications for endoscopic surgery were as follows: 1) the presence of symptoms; 2) age older than 70 years; 3 ) absence of moderate or massive brain contusion/

ABBREVIATIONS GCS = Glasgow Coma Scale; $m R S=$ modified Rankin Scale; $\mathrm{SDH}=$ subdural hematoma.

SUBMITTED April 30, 2014. ACCEPTED October 17, 2014.

INCLUDE WHEN CITING Published online April 24, 2015; DOI: 10.3171/2014.10.JNS14915.

DISCLOSURE The authors report no conflict of interest concerning the materials or methods used in this study or the findings specified in this paper. 


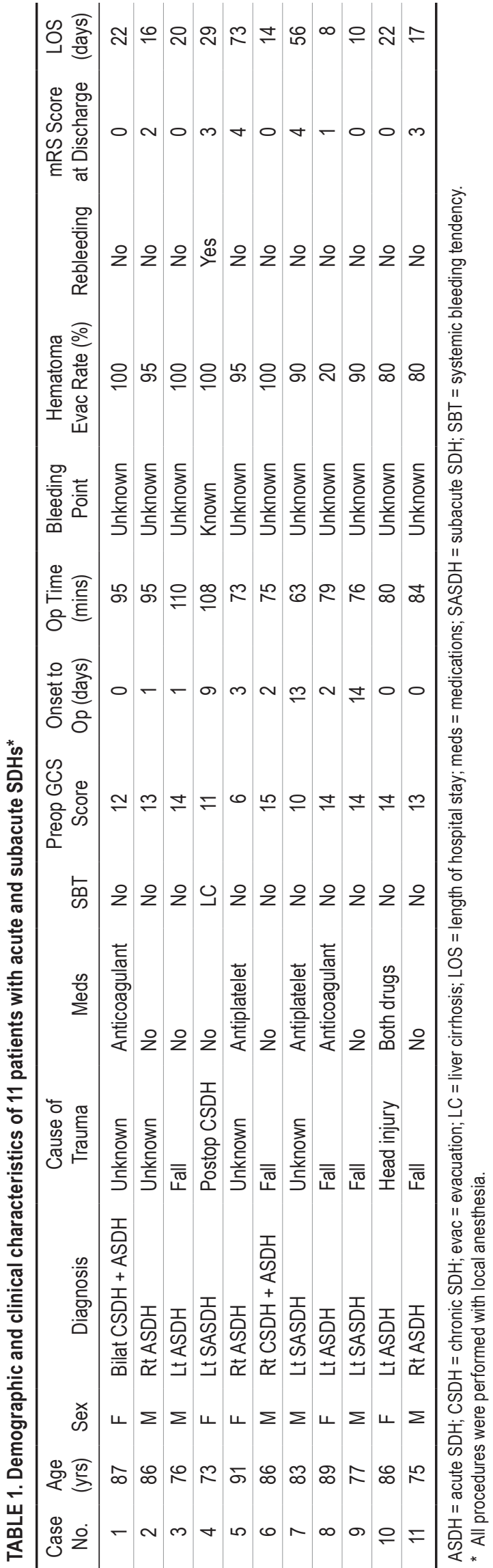

hematoma; 4) absence of an enlarging SDH; and 5) no high risk of bleeding (high risk of bleeding was defined as thrombocytopenia $\left[<5 \times 10^{4} \mu \mathrm{l}\right]$ or disseminated intravascular coagulation).

In 3 patients surgery was performed on the 1st day, whereas the remaining 8 patients underwent surgery 1-14 days after onset.

\section{Endoscopic Procedure}

All surgery was performed under local anesthesia. A bur hole was made just above the center of the hematoma; the hole was enlarged to approximately $2-3 \mathrm{~cm}$ and the bone edge was resected diagonally, so that surgical instruments could be inserted through it and moved widely (Fig. 1A). The dura mater was opened, and a 4-mm rigid endoscope with a $0^{\circ}$ lens and malleable irrigation suction cannula were inserted (Fig. 1B and C). The malleable irrigation suction cannula that we developed is a very useful instrument; this suction cannula is able to twist to various angles (Fig. 1C). When this suction is connected to an electrocauterizer, it also functions as a monopolar electrocautery probe. Additionally, it is coated with polytetrafluoroethylene to prevent the insulating coating near the edge of the bur hole from peeling off.

The rigid endoscope was held in the right hand, while a suction cannula was held in the left hand. If the hematoma was hard, it was removed by forceps. When bleeding from a vessel on the brain surface was seen, a suction cannula was placed at the bleeding point, and the bleeding vessel was coagulated. The subdural space was copiously irrigated with artificial CSF (ARTCEREB; Otsuka Pharmaceutical Co., Ltd.), after which bleeding from the brain surface was confirmed to have stopped. The dura mater was closed and covered with appropriately sized pieces of Surgicel (Johnson \& Johnson) to fill the dead space of the bur hole. A drainage tube was not inserted in the subdural space. Head skin was closed with 3-0 Vicryl Rapide sutures (Ethicon, Johnson \& Johnson).

Informed consent for the procedure was obtained from all patients' families. This method was approved by the ethics screening committee of our institution.

\section{Results}

Endoscopic surgery was performed under local anesthesia in all patients. The mean age of the patients was 82.6 years (range 73-91 years). There were 5 female and 6 male patients. The locations of SDHs were as follows: 4 right side, 6 left side, and 1 bilateral. The mean preoperative GCS score was 12 . No surgery-related mortality occurred, and rebleeding was noted in only 1 patient (9.1\%). This patient (Case 4) underwent reoperation by endoscopic surgery, and the bleeding point was seen and subsequently coagulated by our coagulation system. The modified Rankin Scale (mRS) score in this case at discharge was 3 . Five patients were receiving antithrombotic therapy at admission, but no rebleeding after endoscopic surgery was observed in these patients. The mean operation time was $85.3 \pm 14.9$ minutes. The mean hematoma evacuation rate was $87 \% \pm 23.2 \%$. One patient (Case 8 ) had a low evacuation rate because the hematoma was very hard. Neverthe- 

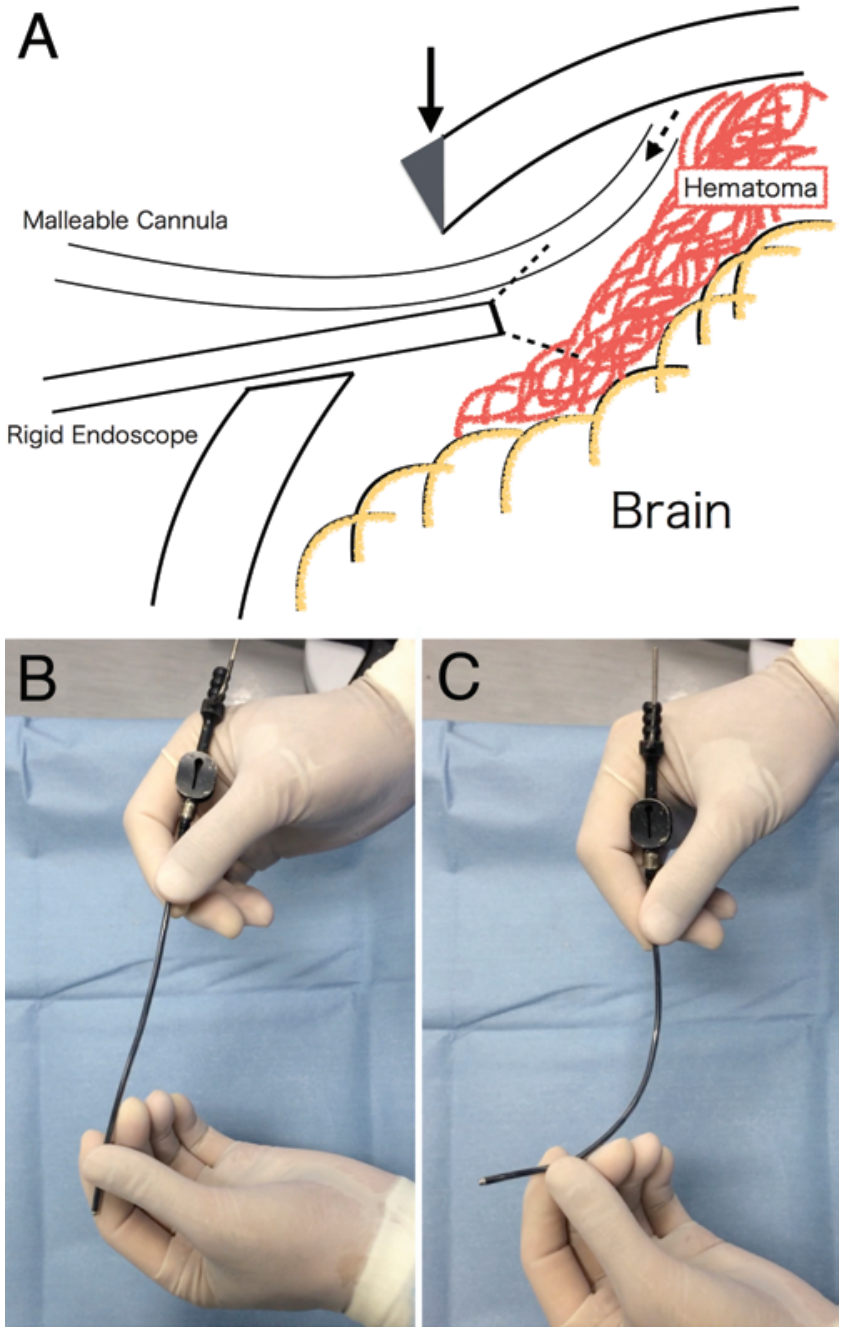

FIG. 1. A: Intraoperative schema showing the procedure in which the rigid endoscope and the malleable suction are used. The bone edge was resected aslant, so that surgical instruments could be inserted through it and moved widely (arrow). Copyright Kimihiko Yokosuka. Published with permission. B: Combined irrigation-coagulation suction for endoscopic evacuation of an intracranial hematoma. C: This suction cannula is able to twist to various angles.

less, after surgery this patient's symptoms improved, and the mRS score was 1 at discharge. The mean hospitalization period was $26 \pm 20.2$ days. At discharge, 7 patients (63.6\%) had a good recovery (mRS Score $0-2$ ).

\section{Illustrative Cases}

\section{Case 3}

Acute SDH

A 76-year-old man presented to our hospital with headache and vomiting after a fall. On admission, his level of consciousness was E4V4M6 on the GCS. Initial head CT revealed a left acute SDH without contusion or other intracranial hematoma. The acute SDH was approximately $1 \mathrm{~cm}$ thick. No abnormalities were found on laboratory analysis. The patient's medical history was unremarkable. He was initially treated conservatively because his clinical symptoms were not severe. After 12 hours of observation,
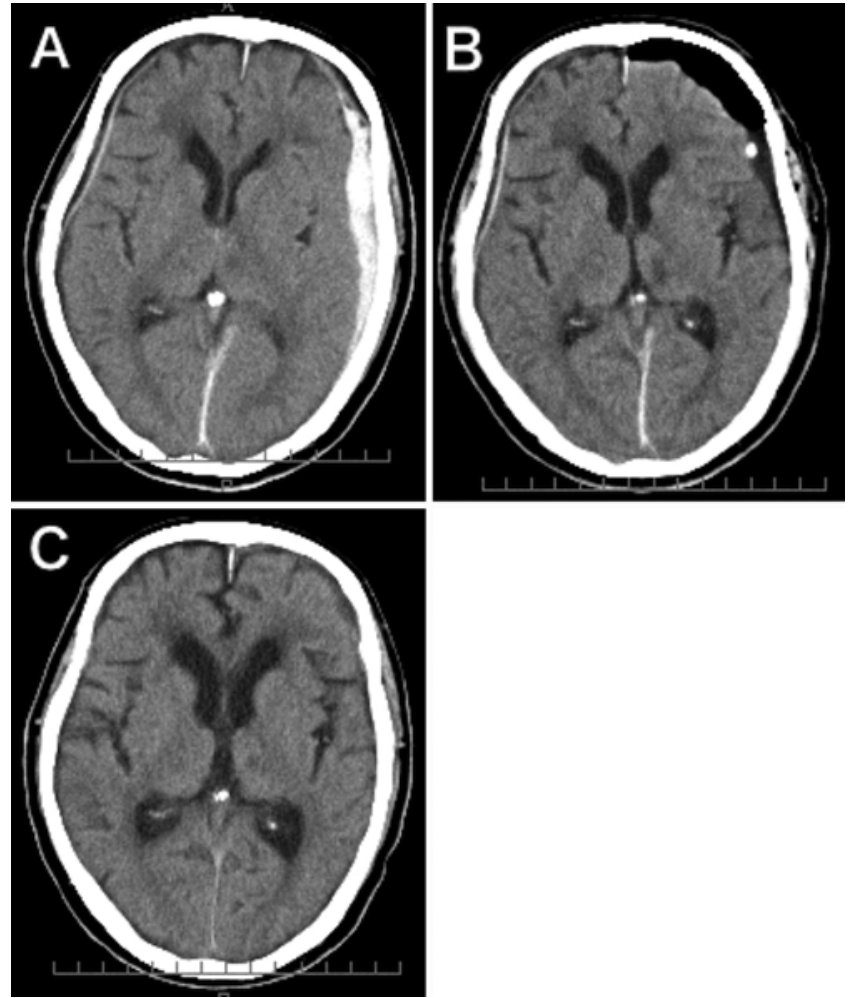

FIG. 2. Case 3. Illustrative case of a patient with a left acute SDH. A: Preoperative noncontrast CT scan showing a left acute SDH. B: One day after endoscopic surgery, CT scan showing complete evacuation of SDH. C: Three months after endoscopic surgery, CT scan showing no recurrence.

no enlargement of the hematoma was seen on CT (Fig. $2 \mathrm{~A}$ ), but aphasia and right hemiparesis appeared. Therefore, endoscopic evacuation of the hematoma was performed. The hematoma was removed easily using the suction cannula (Fig. 3 upper), and there was no injured blood vessel on the brain surface (Fig. 3 lower). The postoperative CT demonstrated complete hematoma removal (Fig. 2B). After surgery, the patient became alert, and the aphasia and right hemiparesis were improved. At discharge on postoperative Day 20, his mRS score was 0. Recurrence was not observed at 3 months after surgery, and the patient returned to normal activities of daily living (Fig. 2C).

\section{Case 9}

\section{Subacute SDH}

A 77-year-old man was referred to our hospital by a doctor at a local hospital for treatment of subacute SDH. On admission he was alert, and the subacute SDH was approximately $1 \mathrm{~cm}$ thick on the initial CT (Fig. 4A). No abnormalities were found on laboratory analyses, and his medical history was unremarkable. Conservative treatment was continued because his only symptom was mild headache. The patient was discharged from our hospital 13 days after admission with no clinical symptoms. However, on the next day the patient was readmitted because of sudden-onset aphasia. A CT scan showed the left subacute SDH (Fig. 4B), and evacuation of the lesion by endoscope was performed. The postoperative CT scan demonstrated 

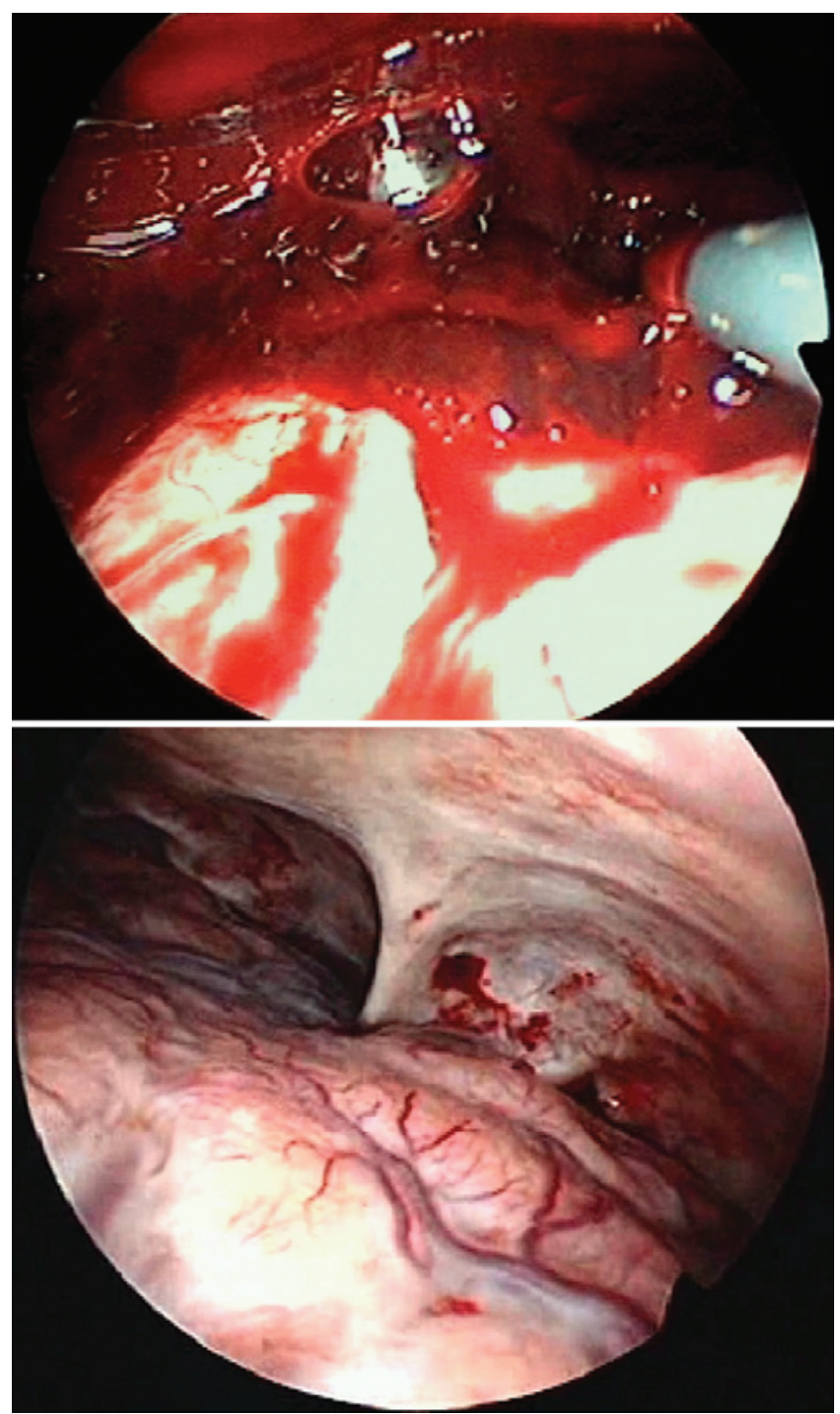

FIG. 3. Case 3. Upper: Intraoperative photograph showing a solid clot located between the dura mater and the brain surface. Using combined irrigation-coagulation suction, the hematoma is evacuated. Lower: Intraoperative photograph showing that after hematoma evacuation there is no injured blood vessel on the brain surface.

subtotal hematoma removal (Fig. 4C). After surgery, the patient's aphasia was improved. At the time of discharge on postoperative Day 10, his mRS score was 0. Recurrence was not observed at 3 months after surgery, and the patient returned to normal activities of daily living (Fig. 4D).

\section{Discussion}

Along with the aging of the Japanese population, the number of elderly patients with acute SDH caused by mild head trauma has been increasing. ${ }^{7}$ Furthermore, most elderly patients with acute SDH initially received conservative treatment, and the SDH remained through the subacute phase..$^{5}$ Subacute $\mathrm{SDH}$ has been reported to occur in approximately $10 \%$ of acute SDH cases treated conservatively. ${ }^{4,13}$ Recently, minimally invasive surgery with a rigid
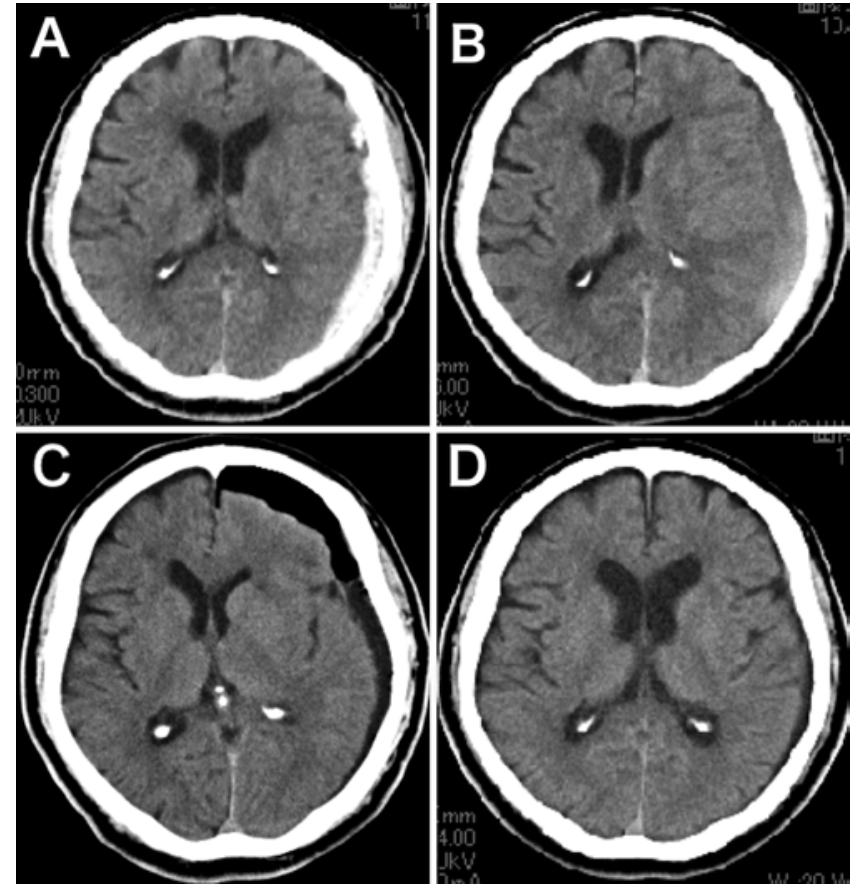

FIG. 4. Case 9. Illustrative case of a patient with a left subacute SDH. A: Initial CT scan showing a left subacute SDH, with no injury in the cerebral parenchyma. B: Second CT scan obtained 13 days after onset showing an increase in hematoma volume with midline shift and a change to low density. C: One day after endoscopic surgery, CT scan showing subtotal evacuation of the SDH. D: Three months after endoscopic surgery, CT scan showing no recurrence.

or flexible endoscope for chronic SDH has been successfully demonstrated..$^{2,3,9,11,14}$ In a few previous reports bur hole surgery was performed for subacute SDH, with good outcomes. ${ }^{5,6,16}$ Yamada et al. reported on 20 patients in whom subacute SDH was treated with bur hole surgery. ${ }^{16}$ Eighteen patients underwent surgery once, with no recurrence, and good recovery was achieved in $77.8 \%$ of cases. However, endoscopic methods were not used.

Karakhan and Khodnevich previously reported bur hole surgery performed using a flexible endoscope for traumatic intracranial hemorrhages. ${ }^{8}$ The mortality rate in 122 patients with SDH who underwent endoscopic surgery was $16.4 \%$. There were 26 patients with acute SDH, 47 with subacute SDH, and 49 with chronic SDH. However, these authors examined only the mortality and rebleeding rates after their surgeries, not functional outcomes.

Recently, Codd et al. ${ }^{1}$ reported that they performed endoscopic surgery using a rigid endoscope for a patient with acute SDH, and that this patient had a good outcome. We performed endoscopic surgery using a rigid endoscope for 11 patients with acute and subacute SDHs, and they also had good outcomes. To the best of our knowledge, this is the first report of endoscopic surgery performed using a rigid endoscope in more than 10 patients with acute and subacute SDHs. These surgeries showed good outcomes due to the careful selection of the patients.

It is necessary to choose surgical indications carefully because, compared with craniotomy or craniectomy for patients with acute and subacute SDHs, endoscopic sur- 
gery is unable to decompress brain swelling. Karakhan and Khodnevich ${ }^{8}$ reported the contraindications of endoscopic surgery as follows: 1) widespread brain laceration; 2) large bleeding vessel; 3) brain prolapse; and 4) calcification of a hematoma. They also excluded patients needing decompressive craniotomy or craniectomy. The most important point of these indications for endoscopic surgery is safety. Therefore, we decide on endoscopic surgery for acute and subacute SDH based on these contraindications. Our indications for endoscopic surgery are as follows: 1) the presence of symptoms; 2) age older than 70 years; 3) absence of moderate or massive brain contusion/ hematoma; 4) absence of an enlarging $\mathrm{SDH}$; and 5) no high risk of bleeding. Using these criteria, we performed endoscopic surgery in 11 patients with acute and subacute SDH. In 2 patients (Cases 5 and 7) the mRS score was 4 at discharge. As a cause of poor outcome, it was thought that the preoperative GCS score was lower in the poor than in the good outcome group. Rebleeding was observed in 1 case. This patient had a confirmed bleeding point, but coagulation was insufficient. Repeat endoscopic surgery was done, the bleeding point was completely coagulated, and there was no further recurrence. On the other hand, patients receiving antithrombotic drugs (antiplatelet drugs and/or anticoagulants) showed no rebleeding. This finding suggests that endoscopic surgery for acute and subacute SDH is safe even for patients with a high risk of bleeding.

The mean hematoma evacuation rate was $87 \% \pm 23.2 \%$. One patient had a low evacuation rate, but her mRS score was 1 at discharge. We conclude that a decrease of intracranial pressure and a decompression of brain are important aspects of this surgery. Therefore, we conclude that complete hematoma evacuation is not necessary.

Another important consideration is the development of useful equipment. Nagasaka et al. ${ }^{12}$ performed combined irrigation-coagulation suction for endoscopic evacuation of intracranial hemorrhage. However, it is difficult to insert the suction cannula that they used into the subdural space, because this cannula cannot twist to various angles. Therefore, we improved the suction cannula and created an irrigation suction cannula with a malleable nozzle (Fig. $1 \mathrm{~B}$ and $\mathrm{C}$ ). This suction cannula can evacuate hematomas that are deeply located or at various angles.

In this study, the patients' mean age was 82.6 years, and there was no mortality and morbidity. Seven of $11 \mathrm{pa}-$ tients $(63.6 \%)$ returned to normal activities of daily life. Therefore, it is thought that endoscopic surgery is useful for elderly patients with acute and subacute SDHs. However, our report is on a small number of cases, and it is a retrospective study. Thus, it will be necessary to observe more cases and to examine long-term outcomes. Also, it will be necessary to evaluate the effectiveness of our procedure compared with large craniotomy/craniectomy for hematoma removal in elderly patients with acute and subacute SDHs.

\section{Conclusions}

Endoscopic hematoma evacuation of acute and subacute SDHs for selected elderly patients is a safe and effective method. We believe that this technique may be a less invasive surgical approach for treating elderly patients with acute and subacute SDHs.

\section{References}

1. Codd PJ, Venteicher AS, Agarwalla PK, Kahle KT, Jho DH: Endoscopic burr hole evacuation of an acute subdural hematoma. J Clin Neurosci 20:1751-1753, 2013

2. Hellwig D, Heinze S, Riegel T, Benes L: Neuroendoscopic treatment of loculated chronic subdural hematoma. Neurosurg Clin N Am 11:525-534, 2000

3. Hellwig D, Kuhn TJ, Bauer BL, List-Hellwig E: Endoscopic treatment of septated chronic subdural hematoma. Surg Neurol 45:272-277, 1996

4. Izumihara A, Orita T, Tsurutani T, Kajiwara K: [Natural course of non-operative cases of acute subdural hematoma: sequential computed tomographic study in the acute and subacute stages.] No Shinkei Geka 25:307-314, 1997 (Jpn)

5. Izumihara A, Yamashita K, Abe T, Ozaki R: [Operative cases of acute subdural hematoma in the subacute stage.] Neurosurg Emerg 15:149-156, 2010 (Jpn)

6. Izumihara A, Yamashita K, Murakami T: Acute subdural hematoma requiring surgery in the subacute or chronic stage. Neurol Med Chir (Tokyo) 53:323-328, 2013

7. Kameyama M, Karibe H, Kawase M, Hayashi T, Hirano T, Tominaga T: [Severe head injury and age jn Japan Neurotrauma Data Bank: comparison among Project 1998, 2004 and 2009.] Neurotraumatology 36:10-16, 2013 (Jpn)

8. Karakhan VB, Khodnevich AA: Endoscopic surgery of traumatic intracranial haemorrhages. Acta Neurochir Suppl 61:84-91, 1994

9. Masopust V, Netuka D, Häckel M: Chronic subdural haematoma treatment with a rigid endoscope. Minim Invasive Neurosurg 46:374-379, 2003

10. McKissock W, Richardson A, Bloom W: Subdural hematoma: a review of 389 cases. Lancet 1:1365-1369, 1960

11. Mobbs R, Khong P: Endoscopic-assisted evacuation of subdural collections. J Clin Neurosci 16:701-704, 2009

12. Nagasaka T, Tsugeno M, Ikeda H, Okamoto T, Takagawa Y, Inao $\mathrm{S}$, et al: Balanced irrigation-suction technique with a multifunctional suction cannula and its application for intraoperative hemorrhage in endoscopic evacuation of intracerebral hematomas: technical note. Neurosurgery 65:E826E827, 2009

13. Okumura Y, Shimomura T, Park YS: [A study of acute subdural hematoma developing into hematoma with capsule formation.] No Shinkei Geka 26:691-698, 1998 (Jpn)

14. Rodziewicz GS, Chuang WC: Endoscopic removal of organized chronic subdural hematoma. Surg Neurol 43:569-573, 1995

15. Wilberger JE Jr, Harris M, Diamond DL: Acute subdural hematoma: morbidity, mortality, and operative timing. J Neurosurg 74:212-218, 1991

16. Yamada T, Natori Y, Yuhi F: [Examination of the clinical course of traumatic subacute subdural hematoma.] Neurosurg Emerg 16:43-48, 2011 (Jpn)

\section{Author Contributions}

Conception and design: Yokosuka. Acquisition of data: Yokosuka. Analysis and interpretation of data: Yokosuka. Administrative/technical/material support: Matsumura, Takai, Hagino, Matsushita, Toi, Matsubara. Study supervision: Uno.

\section{Correspondence}

Kimihiko Yokosuka, Department of Neurosurgery, Kawasaki Medical School, 577 Matsushima, Kurashiki, Okayama 7010192, Japan. email: n.s.hiko@me.com. 